\title{
CLASSICAL ORTHOGONAL POLYNOMIALS AND LEVERRIER-FADDEEV ALGORITHM FOR THE MATRIX PENCILS $s E-A$
}

\author{
JAVIER HERNÁNDEZ AND FRANCISCO MARCELLÁN
}

Received 16 May 2005; Revised 27 March 2006; Accepted 25 April 2006

In this contribution we present an extension of the Leverrier-Faddeev algorithm for the simultaneous computation of the determinant and the adjoint matrix $B(s)$ of a pencil $s E-A$ where $E$ is a singular matrix but $\operatorname{det}(s E-A) \not \equiv 0$. Using a previous result by the authors we express $B(s)$ and $\operatorname{det}(s E-A)$ in terms of classical orthogonal polynomials.

Copyright (c) 2006 Hindawi Publishing Corporation. All rights reserved.

\section{Introduction}

Consider a linear, time-invariant, multivariable singular system described in the state space as follows:

$$
\begin{gathered}
E \dot{x}=A x+B u, \\
y=C x,
\end{gathered}
$$

where $E \in \mathbb{C}^{n \times n}$ is a singular matrix, $x$ is the $n$-dimensional state vector, $u$ is the $m$ dimensional input vector, $y$ is the $r$-dimensional output vector, and $A, B$, and $C$ are matrices with complex entries and appropriate dimension.

We can take the Laplace transform of our system (1.1). If $\operatorname{det}(s E-A) \not 0$, then the following transfer function appears:

$$
H(s)=C(s E-A)^{-1} B
$$

which, in general, is a strictly proper rational matrix (see $[1,5]$ and references therein).

The computation of $(s E-A)^{-1}$ can be carried out by using the Cramer rule, which requires the evaluation of $n^{2}$ determinants of $(n-1) \times(n-1)$ polynomial matrices. Clearly, this is not a practical procedure for large $n$. We will describe an extension of the classical Leverrier-Faddeev algorithm using families of classical orthogonal polynomials following our previous contribution [2] when instead of a singular matrix $E$ we used $I_{n}$. Here we generalize a recent result [6] based on the Chebyshev polynomials, a very 
particular family of classical orthogonal polynomials. Notice that in $[3,5]$ an alternative approach using the canonical basis $\left(x^{n}\right)$ in the linear space of polynomials with complex coefficients was given for linear pencils. Along the paper, we will assume that the pencil $s E-A$ is regular, that is, $\operatorname{det}(s E-A) \not \equiv 0$.

The structure of the manuscript is the following. In Section 2 we summarize our algorithm presented in [2] as well as we introduce the basic background about monic classical orthogonal polynomials. In Section 3 we describe the algorithm to find the adjoint matrix $B(s)$ as well as the determinant of a regular pencil $s E-A$, where $E$ is a singular matrix. We also cover a gap in [6] concerning the connection between $\operatorname{det}(s E-A)$ and the adjoint matrix of $(s E-A)$. Finally, in Section 4 , some numerical examples in order to test our algorithm will be shown.

\section{Leverrier-Faddeev algorithm and classical orthogonal polynomials}

For a matrix $A \in \mathbb{C}^{n \times n}$ an algorithm attributed to Leverrier, Faddeev, and others allows the simultaneous determination of the characteristic polynomial of $A$ and the adjoint matrix of $s I_{n}-A$. As it is shown in [1], if

$$
\begin{gathered}
p_{A}(s)=\operatorname{det}\left(s I_{n}-A\right)=s^{n}+\sum_{k=0}^{n-1} \hat{a}_{n-k} s^{k}, \\
\widetilde{A}(s)=\operatorname{Adj}\left(s I_{n}-A\right)=s^{n-1} I_{n}+\sum_{k=0}^{n-2} s^{k} \widehat{B}_{n-k-1},
\end{gathered}
$$

then the relation between the coefficients $\left(\hat{a}_{k}\right)$ and the matrices $\left(\hat{B}_{k}\right)$ follows by identification of the coefficients of the monomials in the following two equations:

$$
\begin{gathered}
\left(s I_{n}-A\right) \tilde{A}(s)=p_{A}(s) I_{n}, \\
\frac{d p_{A}(s)}{d s}=\operatorname{tr} \tilde{A}(s) .
\end{gathered}
$$

From a numerical point of view, the accuracy of this algorithm is not so good. This is the reason why in [2] we have presented an alternative approach using in (2.1) the representation of $p_{A}(s)$ and $\widetilde{A}(s)$ in terms of a family of monic classical orthogonal polynomials.

The main reason to do it is related to the following fact.

Proposition 2.1 (see [4]). $\left(P_{n}\right)_{n=0}^{\infty}$ is a family of monic classical orthogonal polynomials (Hermite, Laguerre, Jacobi, and Bessel) if and only if there exist sequences of real numbers $\left(r_{n}\right)$ and $\left(s_{n}\right)$ such that

$$
P_{n}(s)=\frac{P_{n+1}^{\prime}(s)}{n+1}+r_{n} \frac{P_{n}^{\prime}(s)}{n}+s_{n} \frac{P_{n-1}^{\prime}(s)}{n-1} \text { for } n \geqslant 2 .
$$

The coefficients that appear in (2.3) are given in Table 2.1.

Notice that the Hermite case appears when $r_{n}=s_{n}=0, n \geqslant 2$. The Laguerre case appears when $s_{n}=0, n \geqslant 2$. Finally, the Jacobi and the Bessel cases are related to the case $s_{n} \neq 0$ for every $n \geqslant 2$. 
TABLe 2.1. Coefficients in the relation of Proposition 2.1.

\begin{tabular}{lcc}
\hline & $r_{n}$ & $s_{n}$ \\
\hline Hermite & 0 & 0 \\
Laguerre & $n$ & 0 \\
Jacobi & $2 n(\alpha-\beta)$ & $-\frac{4 n(n-1)(n+\alpha)(n+\beta)}{(2 n+\alpha+\beta-1)(2 n+\alpha+\beta)^{2}(2 n+\alpha+\beta+1)}$ \\
& $\frac{4 n+\alpha)(2 n+\alpha+\beta+2)}{(2 n+\alpha+\alpha)(2 n+\alpha+2)}$ & $\frac{4 n(n-1)}{(2 n+\alpha-1)(2 n+\alpha)^{2}(2 n+\alpha+1)}$ \\
Bessel & $\frac{(2 n+\alpha)}{2}$ &
\end{tabular}

TABle 2.2. Coefficients in the three-term recurrence relation (2.4).

\begin{tabular}{lcc}
\hline & $\beta_{n}$ & $\gamma_{n}$ \\
\hline Hermite & 0 & $\frac{n}{2}$ \\
Laguerre & $2 n+\alpha+1$ & $n(n+\alpha)$ \\
Jacobi & $\frac{\beta^{2}-\alpha^{2}}{(2 n+\alpha+\beta)(2 n+\alpha+\beta+2)}$ & $\frac{4 n(n+\alpha)(n+\beta)(n+\alpha+\beta)}{(2 n+\alpha+\beta-1)(2 n+\alpha+\beta)^{2}(2 n+\alpha+\beta+1)}$ \\
Bessel & $-\frac{2 \alpha}{(2 n+\alpha)(2 n+\alpha+2)}$ & $-\frac{4 n(n+\alpha)}{(2 n+\alpha-1)(2 n+\alpha)^{2}(2 n+\alpha+1)}$ \\
\hline
\end{tabular}

The second ingredient for our algorithm is the fact that if $\left(P_{n}\right)_{n=0}^{\infty}$ is a family of monic classical orthogonal polynomials, then the following three-term recurrence relation holds:

$$
\begin{gathered}
s P_{n}(s)=P_{n+1}(s)+\beta_{n} P_{n}(s)+\gamma_{n} P_{n-1}(s), \quad n \geqslant 1 \text { with } \gamma_{n} \neq 0, \\
P_{0}(s)=1, \quad P_{1}(s)=s-\beta_{0} .
\end{gathered}
$$

The coefficients that appear in (2.4) are given in Table 2.2.

If we expand the characteristic polynomial $p_{A}(s)$ of $A$ as well as the adjoint matrix $\tilde{A}(s)$ of $s I_{n}-A$ in terms of the above basis of monic classical orthogonal polynomials, that is,

$$
p_{A}(s)=P_{n}(s)+\sum_{k=0}^{n-1} \hat{a}_{n-k} P_{k}(s), \quad \tilde{A}(s)=P_{n-1}(s) I_{n}+\sum_{k=0}^{n-2} P_{k}(s) \hat{B}_{n-k-1},
$$

and take into account (2.2) together with (2.3) and (2.4), then we get the following.

Proposition 2.2 (see [2]). (i) For $k=1, \ldots, n$,

$$
k \hat{a}_{k}=\left(\beta_{n-k}-r_{n-k}\right) \operatorname{tr} \widehat{B}_{k-1}+\left(\gamma_{n-k+1}-s_{n-k+1}\right) \operatorname{tr} \widehat{B}_{k-2}-\operatorname{tr}\left(A \widehat{B}_{k-1}\right)
$$


4 Matrix pencils and classical orthogonal polynomials

Data: $\left\{\beta_{k}\right\}_{k=0}^{n-1},\left\{\gamma_{k}\right\}_{k=1}^{n},\left\{r_{k}\right\}_{k=0}^{n-1},\left\{s_{k}\right\}_{k=1}^{n}$.

Initial Condition: $\widehat{B}_{-1}=0, \widehat{B}_{0}=I_{n}$.

For $k=1,2, \ldots, n-1$

$$
\begin{gathered}
\hat{a}_{k}=(1 / k)\left[\left(\beta_{n-k}-r_{n-k}\right) \operatorname{tr} \widehat{B}_{k-1}+\left(\gamma_{n-k+1}-s_{n-k+1}\right) \operatorname{tr} \widehat{B}_{k-2}-\operatorname{tr}\left(A \hat{B}_{k-1}\right)\right], \\
\hat{B}_{k}=A \hat{B}_{k-1}+\hat{a}_{k} I_{n}-\gamma_{n-k+1} \hat{B}_{k-2}-\beta_{n-k} \hat{B}_{k-1} .
\end{gathered}
$$

End (For)

$$
\hat{a}_{n}=(1 / n)\left[\left(\beta_{0}-r_{0}\right) \operatorname{tr} \widehat{B}_{n-1}+\left(\gamma_{1}-s_{1}\right) \operatorname{tr} \widehat{B}_{n-2}-\operatorname{tr}\left(A \widehat{B}_{n-1}\right)\right]
$$

Algorithm 2.1

(ii) for $k=1,2, \ldots, n-1$,

$$
\widehat{B}_{k}=A \widehat{B}_{k-1}+\hat{a}_{k} I_{n}-\gamma_{n-k+1} \widehat{B}_{k-2}-\beta_{n-k} \widehat{B}_{k-1},
$$

with the convention $\widehat{B}_{-1}=0, r_{0}=0, s_{1}=0$.

Indeed the algorithm to find $\left(a_{k}\right)$ and $\left(B_{k}\right)$ is in Algorithm 2.1.

\section{Regular pencils}

Now, we are interested in the computation of $a(s)=\operatorname{det}(s E-A)$, assuming $s E-A$ is a regular pencil, and $B(s)=\operatorname{Adj}(s E-A)$, where $A, E \in \mathbb{C}^{n \times n}$ and $E$ is a singular matrix. If in the expressions of the previous section we replace $A$ by $A(s)=-s E+A$, then we get

$$
\tilde{a}(\lambda, s):=\operatorname{det}\left(\lambda I_{n}-A(s)\right)=P_{n}(\lambda)+\sum_{k=0}^{n-1} \hat{a}_{n-k}(s) P_{k}(\lambda)
$$

as well as

$$
\widetilde{B}(\lambda, s):=\operatorname{Adj}\left(\lambda I_{n}-A(s)\right)=P_{n-1}(\lambda) I_{n}+\sum_{k=0}^{n-2} P_{k}(\lambda) \widehat{B}_{n-k-1}(s) .
$$

Thus, from (2.6) and (2.7) we get

$$
\begin{aligned}
k \hat{a}_{k}(s)= & \left(\beta_{n-k}-r_{n-k}\right) \operatorname{tr} \hat{B}_{k-1}(s)-\operatorname{tr}\left(A(s) \hat{B}_{k-1}(s)\right) \\
& +\left(\gamma_{n-k+1}-s_{n-k+1}\right) \operatorname{tr} \hat{B}_{k-2}(s), \quad k=1, \ldots, n
\end{aligned}
$$

as well as

$$
\widehat{B}_{k}(s)=\hat{a}_{k}(s) I_{n}-\gamma_{n-k+1} \widehat{B}_{k-2}(s)-\beta_{n-k} \widehat{B}_{k-1}(s)+A(s) \widehat{B}_{k-1}(s)
$$


for $k=1, \ldots, n-1$. Thus, if $\lambda=0$ in (3.1) and (3.2), then we get

$$
\begin{gathered}
a(s):=\operatorname{det}(s E-A)=\tilde{a}(0, s)=P_{n}(0)+\sum_{k=0}^{n-1} \hat{a}_{n-k}(s) P_{k}(0), \\
B(s):=\operatorname{Adj}(s E-A)=\widetilde{B}(0, s)=P_{n-1}(0) I_{n}+\sum_{k=0}^{n-2} P_{k}(0) \hat{B}_{n-k-1}(s) .
\end{gathered}
$$

Taking into account $\operatorname{deg}\left(P_{k}(s)\right)=k$ for all $k \geqslant 0$, (3.3), and (3.4), we can assure that the degrees of the polynomial $\hat{a}_{k}(s), k=1,2, \ldots, n$, and the polynomial matrix $\hat{B}_{k}(s), k=1$, $2, \ldots, n-1$, are at most equal to $k$. Thus for $\hat{a}_{k}(s)$ and $\hat{B}_{k}(s)$ we get the expansions

$$
\begin{gathered}
\hat{a}_{k}(s)=\sum_{j=0}^{k} a_{k, j} P_{j}(s), \quad a_{k, j} \in \mathbb{C}, \\
\hat{B}_{k}(s)=\sum_{j=0}^{k} P_{j}(s) B_{k, j}, \quad B_{k, j} \in \mathbb{C}^{n \times n} .
\end{gathered}
$$

Substituting (3.7) in (3.3), we get

$$
\begin{gathered}
k \sum_{j=0}^{k} a_{k, j} P_{j}(s)=\operatorname{tr}\left(\left(\beta_{n-k}-r_{n-k}\right) \sum_{j=0}^{k-1} P_{j}(s) B_{k-1, j}+\left(\gamma_{n-k+1}-s_{n-k+1}\right) \sum_{j=0}^{k-2} P_{j}(s) B_{k-2, j}\right. \\
\left.+(s E-A) \sum_{j=0}^{k-1} P_{j}(s) B_{k-1, j}\right) .
\end{gathered}
$$

Applying in the right-hand side the three-term recurrence relation, we get

$$
\begin{aligned}
k \sum_{j=0}^{k} a_{k, j} P_{j}(s)= & \operatorname{tr}\left(E B_{k-1, k-1}\right) P_{k}(s) \\
+ & {\left[\left(\beta_{n-k}-r_{n-k}\right) \operatorname{tr} B_{k-1, k-1}+\beta_{k-1} \operatorname{tr}\left(E B_{k-1, k-1}\right)\right.} \\
& \left.\quad-\operatorname{tr}\left(A B_{k-1, k-1}\right)+\operatorname{tr}\left(E B_{k-1, k-2}\right)\right] P_{k-1}(s) \\
+ & \sum_{j=1}^{k-2}\left[\gamma_{j+1} \operatorname{tr}\left(E B_{k-1, j+1}\right)+\beta_{j} \operatorname{tr}\left(E B_{k-1, j}\right)\right. \\
& \quad+\left(\beta_{n-k}-r_{n-k}\right) \operatorname{tr} B_{k-1, j}+\left(\gamma_{n-k+1}-s_{n-k+1}\right) \operatorname{tr} B_{k-2, j} \\
& \left.-\operatorname{tr}\left(A B_{k-1, j}\right)+\operatorname{tr}\left(E B_{k-1, j-1}\right)\right] P_{j}(s) \\
+ & {\left[\gamma_{1} \operatorname{tr}\left(E B_{k-1,1}\right)+\beta_{0} \operatorname{tr}\left(E B_{k-1,0}\right)+\left(\beta_{n-k}-r_{n-k}\right) \operatorname{tr} B_{k-1,0}\right.} \\
& \left.+\left(\gamma_{n-k+1}-s_{n-k+1}\right) \operatorname{tr} B_{k-2,0}-\operatorname{tr}\left(A B_{k-1,0}\right)\right] P_{0}(s) .
\end{aligned}
$$


6 Matrix pencils and classical orthogonal polynomials

Thus, for $k=1,2, \ldots, n$,

$$
\begin{gathered}
k a_{k, 0}=\gamma_{1} \operatorname{tr}\left(E B_{k-1,1}\right)+\beta_{0} \operatorname{tr}\left(E B_{k-1,0}\right)+\left(\beta_{n-k}-r_{n-k}\right) \operatorname{tr} B_{k-1,0} \\
-\operatorname{tr}\left(A B_{k-1,0}\right)+\left(\gamma_{n-k+1}-s_{n-k+1}\right) \operatorname{tr} B_{k-2,0} \\
\vdots \\
k a_{k, j}=\gamma_{j+1} \operatorname{tr}\left(E B_{k-1, j+1}\right)+\beta_{j} \operatorname{tr}\left(E B_{k-1, j}\right)+\operatorname{tr}\left(E B_{k-1, j-1}\right) \\
+\left(\gamma_{n-k+1}-s_{n-k+1}\right) \operatorname{tr} B_{k-2, j}+\left(\beta_{n-k}-r_{n-k}\right) \operatorname{tr} B_{k-1, j} \\
-\operatorname{tr}\left(A B_{k-1, j}\right), \quad j=1, \ldots, k-2 \\
\vdots \\
k a_{k, k-1}=\left(\beta_{n-k}-r_{n-k}\right) \operatorname{tr} B_{k-1, k-1}+\operatorname{tr}\left(E B_{k-1, k-2}\right) \\
+\beta_{k-1} \operatorname{tr}\left(E B_{k-1, k-1}\right)-\operatorname{tr}\left(A B_{k-1, k-1}\right) \\
k a_{k, k}=\operatorname{tr}\left(E B_{k-1, k-1}\right) .
\end{gathered}
$$

In an analogous way, substituting (3.7) in (3.4),

$$
\begin{aligned}
\sum_{j=0}^{k} P_{j}(s) B_{k, j}= & \sum_{j=0}^{k} a_{k, j} P_{j}(s) I_{n}-\gamma_{n-k+1} \sum_{j=0}^{k-2} P_{j}(s) B_{k-2, j} \\
& -\beta_{n-k} \sum_{j=0}^{k-1} P_{j}(s) B_{k-1, j}+(-s E+A) \sum_{j=0}^{k-1} P_{j}(s) B_{k-1, j} .
\end{aligned}
$$

Using again the three-term recurrence relation, we get

$$
\begin{aligned}
\sum_{j=0}^{k} P_{j}(s) B_{k, j}= & P_{k}(s)\left[a_{k, k} I_{n}-E B_{k-1, k-1}\right] \\
& +P_{k-1}(s)\left[a_{k, k-1} I_{n}-E B_{k-1, k-2}+\left(A-\beta_{k-1} E-\beta_{n-k} I_{n}\right) B_{k-1, k-1}\right] \\
& +\sum_{j=1}^{k-2} P_{j}(s)\left[a_{k, j} I_{n}-E B_{k-1, j-1}+\left(A-\beta_{j} E-\beta_{n-k} I_{n}\right) B_{k-1, j}\right. \\
& \left.\quad-\gamma_{j+1} E B_{k-1, j+1}-\gamma_{n-k+1} B_{k-2, j}\right] \\
& +P_{0}(s)\left[a_{k, 0} I_{n}+\left(A-\beta_{0} E-\beta_{n-k} I_{n}\right) B_{k-1,0}\right. \\
& \left.-\gamma_{1} E B_{k-1,1}-\gamma_{n-k+1} B_{k-2,0}\right] .
\end{aligned}
$$


Data: $\left\{\beta_{k}\right\}_{k=0}^{n-1},\left\{\gamma_{k}\right\}_{k=1}^{n},\left\{r_{k}\right\}_{k=0}^{n-1},\left\{s_{k}\right\}_{k=1}^{n}$.

Initial Condition: $B_{i, j}=0$, if $i<j$ or $j<0, a_{0,0}=1, B_{0,0}=I_{n}$.

For $k=1, \ldots, n-1$

$$
\begin{aligned}
& \alpha_{n-k}=\beta_{n-k}-r_{n-k} \text {. } \\
& \delta_{n-k+1}=\gamma_{n-k+1}-s_{n-k+1} \text {. } \\
& A_{k}=A-\beta_{n-k} I_{n} \text {. } \\
& \text { For } j=0,1, \ldots, k \\
& a_{k, j}:=(1 / k)\left[\gamma_{j+1} \operatorname{tr}\left(E B_{k-1, j+1}\right)+\beta_{j} \operatorname{tr}\left(E B_{k-1, j}\right)+\alpha_{n-k} \operatorname{tr} B_{k-1, j}\right. \\
& \left.+\operatorname{tr}\left(E B_{k-1, j-1}\right)+\delta_{n-k+1} \operatorname{tr} B_{k-2, j}-\operatorname{tr}\left(A B_{k-1, j}\right)\right] . \\
& B_{k, j}:=a_{k, j} I_{n}-E B_{k-1, j-1}+\left(A_{k}-\beta_{j} E\right) B_{k-1, j}-\gamma_{j+1} E B_{k-1, j+1} \\
& -\gamma_{n-k+1} B_{k-2, j} \text {. }
\end{aligned}
$$

End (For j).

End (For k).

For $j=0,1, \ldots, n$

$$
\begin{aligned}
a_{n, j}:=(1 / n)\left[\gamma_{j+1} \operatorname{tr}\left(E B_{n-1, j+1}\right)+\beta_{j} \operatorname{tr}\left(E B_{n-1, j}\right)+\beta_{0} \operatorname{tr} B_{n-1, j}\right. \\
+ \\
\left.+\operatorname{tr}\left(E B_{n-1, j-1}\right)+\gamma_{1} \operatorname{tr} B_{n-2, j}-\operatorname{tr}\left(A B_{n-1, j}\right)\right] .
\end{aligned}
$$

End.

Algorithm 3.1

Thus, for $k=1,2, \ldots, n-1$,

$$
\begin{gathered}
B_{k, 0}=a_{k, 0} I_{n}+\left(A-\beta_{0} E-\beta_{n-k} I_{n}\right) B_{k-1,0}-\gamma_{1} E B_{k-1,1}-\gamma_{n-k+1} B_{k-2,0}, \\
\vdots \\
B_{k, j}=a_{k, j} I_{n}-E B_{k-1, j-1}+\left(A-\beta_{j} E-\beta_{n-k} I_{n}\right) B_{k-1, j} \\
-\gamma_{j+1} E B_{k-1, j+1}-\gamma_{n-k+1} B_{k-2, j}, \quad j=1, \ldots, k-2, \\
\vdots \\
B_{k, k-1}=a_{k, k-1} I_{n}-E B_{k-1, k-2}+\left(A-\beta_{k-1} E-\beta_{n-k} I_{n}\right) B_{k-1, k-1}, \\
B_{k, k}=a_{k, k} I_{n}-E B_{k-1, k-1} .
\end{gathered}
$$

As a conclusion, the algorithm for the computation of the coefficients $a_{i, j}$ in (3.5) and $B_{i, j}$ in (3.6) is as in Algorithm 3.1.

Notice that formula (3.10) in [6] is not right as a simple computation shows. Indeed for a regular pencil it is enough to consider the expression of $a(s)$ and $B(s)$ in the example provided in [6, Section 4].

Next we will give the right result. 
8 Matrix pencils and classical orthogonal polynomials

Theorem 3.1. Let $A, E \in \mathbb{C}^{n \times n}, a(s)=\operatorname{det}(s E-A)$, and $B(s)=\operatorname{Adj}(s E-A)$. Then

$$
\frac{d}{d s} a(s)=\operatorname{tr}(E B(s))
$$

Proof. First, assume that $E$ is a nonsingular matrix. Then $s E-A=\left(s I_{n}-A E^{-1}\right) E$ and

$$
\begin{aligned}
\frac{d}{d s} a(s) & =\operatorname{det}(E) \frac{d}{d s}\left(\operatorname{det}\left(s I_{n}-A E^{-1}\right)\right) \\
& =\operatorname{det}(E) \operatorname{tr}\left(\operatorname{Adj}\left(s I_{n}-A E^{-1}\right)\right) \\
& =\operatorname{det}(E) \operatorname{det}(E)^{-1} \operatorname{det}(s E-A) \operatorname{tr}\left(E(s E-A)^{-1}\right) \\
& =\operatorname{det}(s E-A) \operatorname{tr}\left(E(s E-A)^{-1}\right) \\
& =\operatorname{tr}(E B(s))
\end{aligned}
$$

Next, if $E$ is a singular matrix, then consider $\varepsilon>0$, such that $\varepsilon<\min \left\{\left|\lambda_{i}\right|: \lambda_{i}\right.$ is an eigenvalue of $\left.E, \lambda_{i} \neq 0\right\}$.

Then $E_{\varepsilon}:=E+\varepsilon I_{n}$ is a nonsingular matrix. Using the first part of the proof,

$$
\frac{d}{d s} a_{\varepsilon}(s)=\operatorname{tr}\left(E_{\varepsilon} B_{\varepsilon}(s)\right)
$$

where $a_{\varepsilon}(s)=\operatorname{det}\left(s E_{\varepsilon}-A\right)$ and $B_{\varepsilon}(s):=\operatorname{Adj}\left(s E_{\varepsilon}-A\right)$.

Taking into account $E_{\varepsilon} \rightarrow E, a_{\varepsilon}(s) \rightarrow a(s)$, and $B_{\varepsilon}(s) \rightarrow B(s)$, when $\varepsilon \rightarrow 0$, we deduce our statement.

\section{Examples}

Let $A, E \in \mathbb{C}^{3 \times 3}$ given by

$$
A=\left[\begin{array}{lll}
1 & 1 & 1 \\
1 & 1 & 1 \\
1 & 1 & 1
\end{array}\right], \quad E=\left[\begin{array}{lll}
1 & 0 & 0 \\
0 & 1 & 0 \\
0 & 0 & 0
\end{array}\right]
$$

Notice that $\operatorname{rank} E=2$. It is straightforward to prove that

$$
\begin{gathered}
a(s)=\operatorname{det}(s E-A)=-s^{2}, \\
B(s)=\operatorname{Adj}(s E-A)=\left[\begin{array}{ccc}
-s & 0 & s \\
0 & -s & s \\
s & s & s^{2}-2 s
\end{array}\right] .
\end{gathered}
$$


Applying the algorithm of the previous section for Hermite polynomials $\left\{H_{k}(s)\right\}_{k=0}^{n}$, we get

$$
\begin{aligned}
& a_{1,0}=-\operatorname{tr} A=-3, \quad a_{1,1}=\operatorname{tr} E=2 ; \\
& B_{1,0}=a_{1,0} I_{3}+A=\left[\begin{array}{ccc}
-2 & 1 & 1 \\
1 & -2 & 1 \\
1 & 1 & -2
\end{array}\right], \quad B_{1,1}=a_{1,1} I_{3}-E=\left[\begin{array}{lll}
1 & 0 & 0 \\
0 & 1 & 0 \\
0 & 0 & 2
\end{array}\right] \text {; } \\
& a_{2,0}=\frac{1}{2}\left[\frac{1}{2} \operatorname{tr}\left(E B_{1,1}\right)-\operatorname{tr}\left(A B_{1,0}\right)+3\right]=2, \\
& a_{2,1}=\frac{1}{2}\left[\operatorname{tr}\left(E B_{1,0}\right)-\operatorname{tr}\left(A B_{1,1}\right)\right]=-4, \\
& a_{2,2}=\frac{1}{2} \operatorname{tr}\left(E B_{1,1}\right)=1 ; \\
& B_{2,0}=a_{2,0} I_{3}+A B_{1,0}-\frac{1}{2} E B_{1,1}-I_{3}=\left[\begin{array}{ccc}
\frac{1}{2} & 0 & 0 \\
0 & \frac{1}{2} & 0 \\
0 & 0 & 1
\end{array}\right] \text {, } \\
& B_{2,1}=a_{2,1} I_{3}+A B_{1,1}-E B_{1,0}=\left[\begin{array}{ccc}
-1 & 0 & 1 \\
0 & -1 & 1 \\
1 & 1 & -2
\end{array}\right] \text {, } \\
& B_{2,2}=a_{2,2} I_{3}-E B_{1,1}=\left[\begin{array}{ccc}
0 & 0 & 0 \\
0 & 0 & 0 \\
0 & 0 & 1
\end{array}\right] \\
& a_{3,0}=\frac{1}{3}\left[\frac{1}{2} \operatorname{tr}\left(E B_{2,1}\right)-\operatorname{tr}\left(A B_{2,0}\right)+\frac{1}{2} \operatorname{tr} B_{1,0}\right]=-2, \\
& a_{3,1}=\frac{1}{3}\left[\operatorname{tr}\left(E B_{2,2}\right)-\operatorname{tr}\left(A B_{2,1}\right)+\operatorname{tr}\left(E B_{2,0}\right)+\frac{1}{2} \operatorname{tr} B_{1,1}\right]=1 \text {, } \\
& a_{3,2}=\frac{1}{3}\left[\operatorname{tr}\left(E B_{2,1}\right)-\operatorname{tr}\left(A B_{2,2}\right)\right]=-1, \\
& a_{3,3}=\frac{1}{3} \operatorname{tr}\left(E B_{2,2}\right)=0 .
\end{aligned}
$$

Thus

$$
\begin{gathered}
\hat{a}_{1}(s)=a_{1,0} H_{0}(s)+a_{1,1} H_{1}(s)=-3 H_{0}(s)+2 H_{1}(s), \\
\hat{a}_{2}(s)=a_{2,0} H_{0}(s)+a_{2,1} H_{1}(s)+a_{2,2} H_{2}(s)=2 H_{0}(s)-4 H_{1}(s)+H_{2}(s), \\
\hat{a}_{3}(s)=a_{3,0} H_{0}(s)+a_{3,1} H_{1}(s)+a_{3,2} H_{2}(s)+a_{3,3} H_{3}(s)=-2 H_{0}(s)+H_{1}(s)-H_{2}(s) ; \\
\hat{B}_{1}(s)=H_{0}(s) B_{1,0}+H_{1}(s) B_{1,1}, \\
\hat{B}_{2}(s)=H_{0}(s) B_{2,0}+H_{1}(s) B_{2,1}+H_{2}(s) B_{2,2} .
\end{gathered}
$$


Now, the determinant $a(s)$ and the adjoint $B(s)$ of $s E-A$ are given by

$$
\begin{aligned}
a(s) & =H_{3}(0)+\hat{a}_{1}(s) H_{2}(0)+\hat{a}_{2}(s) H_{1}(0)+\hat{a}_{3}(s) H_{0}(0) \\
& =-\frac{1}{2} \hat{a}_{1}(s)+\hat{a}_{3}(s)=-H_{2}(s)-\frac{1}{2} H_{0}(s), \\
B(s) & =H_{2}(0) \hat{B}_{0}(s)+H_{1}(0) \hat{B}_{1}(s)+H_{0}(0) \hat{B}_{2}(s)=-\frac{1}{2} I_{3}+\hat{B}_{2}(s) \\
& =H_{0}(s)\left[-\frac{1}{2} I_{3}+B_{2,0}\right]+H_{1}(s) B_{2,1}+H_{2}(s) B_{2,2} .
\end{aligned}
$$

Next, applying the algorithm for the family $\left\{L_{k}^{\alpha}(s)\right\}_{k=0}^{n}$ (Laguerre polynomials with parameter $\alpha$ ), we get

$$
\begin{aligned}
& a_{1,0}=(1+\alpha) \operatorname{tr} E+3(3+\alpha)-\operatorname{tr} A=8+5 \alpha, \quad a_{1,1}=\operatorname{tr} E=2 ; \\
& B_{1,0}=\left(a_{1,0}-5-\alpha\right) I_{3}+A-(1+\alpha) E=\left[\begin{array}{ccc}
3+3 \alpha & 1 & 1 \\
1 & 3+3 \alpha & 1 \\
1 & 1 & 4+4 \alpha
\end{array}\right] \text {, } \\
& B_{1,1}=a_{1,1} I_{3}-E=\left[\begin{array}{ccc}
1 & 0 & 0 \\
0 & 1 & 0 \\
0 & 0 & 2
\end{array}\right] \\
& a_{2,0}=\frac{1}{2}\left[(1+\alpha)\left(\operatorname{tr}\left(E B_{1,1}\right)+\operatorname{tr}\left(E B_{1,0}\right)\right)+(2+\alpha)\left(\operatorname{tr} B_{1,0}+6\right)-\operatorname{tr}\left(A B_{1,0}\right)\right] \\
& =4(1+\alpha)(3+2 \alpha) \text {, } \\
& a_{2,1}=\frac{1}{2}\left((2+\alpha) \operatorname{tr} B_{1,1}+\operatorname{tr}\left(E B_{1,0}\right)+(3+\alpha) \operatorname{tr}\left(E B_{1,1}\right)-\operatorname{tr}\left(A B_{1,1}\right)\right)=8+6 \alpha, \\
& a_{2,2}=\frac{1}{2} \operatorname{tr}\left(E B_{1,1}\right)=1 \text {; } \\
& B_{2,0}=\left(a_{2,0}-4-2 \alpha\right) I_{3}+\left(A-(1+\alpha) E-(3+\alpha) I_{3}\right) B_{1,0}-(1+\alpha) E B_{1,1} \\
& =(1+\alpha)\left[\begin{array}{ccc}
2 \alpha & 1 & 2 \\
1 & 2 \alpha & 2 \\
2 & 2 & 2+4 \alpha
\end{array}\right]
\end{aligned}
$$$$
B_{2,1}=a_{2,1} I_{3}+E B_{1,0}+\left(A-(3+\alpha)\left(E+I_{3}\right)\right) B_{1,1}=\left[\begin{array}{ccc}
\alpha & 0 & 1 \\
0 & \alpha & 1 \\
1 & 1 & 4+4 \alpha
\end{array}\right] \text {, }
$$ 


$$
\begin{aligned}
B_{2,2}= & a_{2,2} I_{3}-E B_{1,1}=\left[\begin{array}{lll}
0 & 0 & 0 \\
0 & 0 & 0 \\
0 & 0 & 1
\end{array}\right] \\
a_{3,0}= & \frac{1}{3}\left[(1+\alpha)\left(\operatorname{tr}\left(E B_{2,1}\right)+\operatorname{tr}\left(E B_{2,0}\right)+\operatorname{tr} B_{2,0}+\operatorname{tr} B_{1,0}\right)-\operatorname{tr}\left(A B_{2,0}\right)\right] \\
= & 2 \alpha(1+\alpha)(3+2 \alpha) \\
a_{3,1}= & \frac{1}{3}\left(2(2+\alpha) \operatorname{tr}\left(E B_{2,2}\right)+(3+\alpha) \operatorname{tr}\left(E B_{2,1}\right)+\operatorname{tr}\left(E B_{2,0}\right)++(1+\alpha) \operatorname{tr} B_{2,1}\right. \\
& \left.+(1+\alpha) \operatorname{tr} B_{1,1}-\operatorname{tr}\left(A B_{2,1}\right)\right)=2 \alpha(3+2 \alpha), \\
a_{3,2}= & \frac{1}{3}\left((1+\alpha) \operatorname{tr} B_{2,2}+\operatorname{tr}\left(E B_{2,1}\right)+(5+\alpha) \operatorname{tr}\left(E B_{2,2}\right)-\operatorname{tr}\left(A B_{2,2}\right)\right)=\alpha, \\
a_{3,3}= & \frac{1}{3} \operatorname{tr}\left(E B_{2,2}\right)=0
\end{aligned}
$$

Thus

$$
\begin{aligned}
\hat{a}_{1}(s) & =a_{1,0} L_{0}^{\alpha}(s)+a_{1,1} L_{1}^{\alpha}(s)=(8+5 \alpha) L_{0}^{\alpha}(s)+2 L_{1}^{\alpha}(s), \\
\hat{a}_{2}(s) & =a_{2,0} L_{0}^{\alpha}(s)+a_{2,1} L_{1}^{\alpha}(s)+a_{2,2} L_{2}^{\alpha}(s) \\
& =4(1+\alpha)(3+2 \alpha) L_{0}^{\alpha}(s)+(8+6 \alpha) L_{1}^{\alpha}(s)+L_{2}^{\alpha}(s), \\
\hat{a}_{3}(s) & =a_{3,0} L_{0}^{\alpha}(s)+a_{3,1} L_{1}^{\alpha}(s)+a_{3,2} L_{2}^{\alpha}(s)+a_{3,3} L_{3}^{\alpha}(s) \\
& =2 \alpha(1+\alpha)(3+2 \alpha) L_{0}^{\alpha}(s)+2 \alpha(3+2 \alpha) L_{1}^{\alpha}(s)+\alpha L_{2}^{\alpha}(s) ; \\
\hat{B}_{1}(s) & =L_{0}^{\alpha}(s) B_{1,0}+L_{1}^{\alpha}(s) B_{1,1}, \\
\hat{B}_{2}(s) & =L_{0}^{\alpha}(s) B_{2,0}+L_{1}^{\alpha}(s) B_{2,1}+L_{2}^{\alpha}(s) B_{2,2} .
\end{aligned}
$$

The determinant $a(s)$ and the adjoint $B(s)$ of $s E-A$ are given by

$$
\begin{aligned}
a(s)= & L_{3}^{\alpha}(0)+\hat{a}_{1}(s) L_{2}^{\alpha}(0)+\hat{a}_{2}(s) L_{1}^{\alpha}(0)+\hat{a}_{3}(s) L_{0}^{\alpha}(0) \\
= & -(1+\alpha)(2+\alpha) L_{0}^{\alpha}(s)-2(2+\alpha) L_{1}^{\alpha}(s)-L_{2}^{\alpha}(s), \\
B(s)= & L_{2}^{\alpha}(0) \hat{B}_{0}(s)+L_{1}^{\alpha}(0) \hat{B}_{1}(s)+L_{0}^{\alpha}(0) \hat{B}_{2}(s) \\
= & L_{0}^{\alpha}(s)\left[(1+\alpha)(2+\alpha) I_{3}-(1+\alpha) B_{1,0}+B_{2,0}\right] \\
& +L_{1}^{\alpha}(s)\left[-(1+\alpha) B_{1,1}+B_{2,1}\right]+L_{2}^{\alpha}(s) B_{2,2} .
\end{aligned}
$$


12 Matrix pencils and classical orthogonal polynomials

Finally, if we consider the family $\left\{T_{k}(s)\right\}_{k=0}^{n}$ of the Chebyshev polynomials of first kind, applying the algorithm we get

$$
\begin{aligned}
& a_{1,0}=-\operatorname{tr} A=-3, \quad a_{1,1}=\operatorname{tr} E=2 ; \\
& B_{1,0}=a_{1,0} I_{3}+A=\left[\begin{array}{ccc}
-2 & 1 & 1 \\
1 & -2 & 1 \\
1 & 1 & -2
\end{array}\right], \quad B_{1,1}=a_{1,1} I_{3}-E=\left[\begin{array}{lll}
1 & 0 & 0 \\
0 & 1 & 0 \\
0 & 0 & 2
\end{array}\right] \text {; } \\
& a_{2,0}=\frac{1}{2}\left(\frac{1}{4} \operatorname{tr}\left(E B_{1,1}\right)-\operatorname{tr}\left(A B_{1,0}\right)+\frac{3}{2}\right)=\frac{5}{4} \text {, } \\
& a_{2,1}=\frac{1}{2}\left(\operatorname{tr}\left(E B_{1,0}\right)-\operatorname{tr}\left(A B_{1,1}\right)\right)=-4, \quad a_{2,2}=\frac{1}{2}\left(\operatorname{tr}\left(E B_{1,1}\right)\right)=1 ; \\
& B_{2,0}=a_{2,0} I_{3}+A B_{1,0}-\frac{1}{4} E B_{1,1}-\frac{1}{4} I_{3}=\left[\begin{array}{ccc}
\frac{1}{2} & 0 & 0 \\
0 & \frac{1}{2} & 0 \\
0 & 0 & 1
\end{array}\right], \\
& B_{2,1}=a_{2,1} I_{3}-E B_{1,0}+A B_{1,1}=\left[\begin{array}{ccc}
-1 & 0 & 1 \\
0 & -1 & 1 \\
1 & 1 & -2
\end{array}\right] \text {, } \\
& B_{2,2}=a_{2,2} I_{3}-E B_{1,1}=\left[\begin{array}{ccc}
0 & 0 & 0 \\
0 & 0 & 0 \\
0 & 0 & 1
\end{array}\right] \text {; } \\
& a_{3,0}=\frac{1}{3}\left(\frac{1}{4} \operatorname{tr}\left(E B_{2,1}\right)+\frac{1}{2} \operatorname{tr} B_{1,0}-\operatorname{tr}\left(A B_{2,0}\right)\right)=-2, \\
& a_{3,1}=\frac{1}{3}\left(\frac{1}{4} \operatorname{tr}\left(E B_{2,2}\right)+\operatorname{tr}\left(E B_{2,0}\right)+\frac{1}{2} \operatorname{tr} B_{1,1}-\operatorname{tr}\left(A B_{2,1}\right)\right)=1 \text {, } \\
& a_{3,2}=\frac{1}{3}\left(\operatorname{tr}\left(E B_{2,1}\right)-\operatorname{tr}\left(A B_{2,2}\right)\right)=-1, \\
& a_{3,3}=\frac{1}{3} \operatorname{tr}\left(E B_{2,2}\right)=0 .
\end{aligned}
$$

Thus

$$
\begin{gathered}
\hat{a}_{1}(s)=a_{1,0} T_{0}(s)+a_{1,1} T_{1}(s)=-3 T_{0}(s)+2 T_{1}(s), \\
\hat{a}_{2}(s)=a_{2,0} T_{0}(s)+a_{2,1} T_{1}(s)+a_{2,2} T_{2}(s)=\frac{5}{4} T_{0}(s)-4 T_{1}(s)+T_{2}(s), \\
\hat{a}_{3}(s)=a_{3,0} T_{0}(s)+a_{3,1} T_{1}(s)+a_{3,2} T_{2}(s)+a_{3,3} T_{3}(s)=-2 T_{0}(s)+T_{1}(s)-T_{2}(s) ; \\
\hat{B}_{1}(s)=T_{0}(s) B_{1,0}+T_{1}(s) B_{1,1}, \hat{B}_{2}(s)=T_{0}(s) B_{2,0}+T_{1}(s) B_{2,1}+T_{2}(s) B_{2,2} .
\end{gathered}
$$


The determinant $a(s)$ and the adjoint $B(s)$ of $s E-A$ are given by

$$
\begin{aligned}
a(s) & =T_{3}(0)+\hat{a}_{1}(s) T_{2}(0)+\hat{a}_{2}(s) T_{1}(0)+\hat{a}_{3}(s) T_{0}(0) \\
& =-\frac{1}{2} T_{0}(s)-T_{2}(s) \\
B(s) & =T_{2}(0) \hat{B}_{0}(s)+T_{1}(0) \hat{B}_{1}(s)+T_{0}(0) \hat{B}_{2}(s) \\
& =T_{0}(s)\left(B_{2,0}-\frac{1}{2} I_{3}\right)+T_{1}(s) B_{2,1}+T_{2}(s) B_{2,2} .
\end{aligned}
$$

\section{Acknowledgments}

We thank the comments and suggestions by the anonymous referees in order to improve the presentation of the manuscript. The work of the second author (Francisco Marcellán) has received the financial support from Dirección General de Investigación (Ministerio de Educación y Ciencia) of Spain, Grant BFM2003-06335-C03-02, and INTAS Research Network NeCCA INTAS 03-51-6637. The work of the first author (Javier Hernández) has been supported by Fundación Universidad Carlos III de Madrid.

\section{References}

[1] S. Barnett, Leverrier's algorithm for orthogonal polynomial bases, Linear Algebra and Its Applications 236 (1996), 245-263.

[2] J. Hernández, F. Marcellán, and C. Rodríguez, Leverrier-Fadeev algorithm and classical orthogonal polynomials, Revista Academia Colombiana de Ciencias Exactas, Físicas y Naturales 28 (2004), no. 106, 39-47.

[3] F. L. Lewis, Further remarks on the Cayley-Hamilton theorem and Leverrier's method for the matrix pencil sE - A, IEEE Transactions on Automatic Control 31 (1986), no. 9, 869-870.

[4] F. Marcellán, A. Branquinho, and J. Petronilho, Classical orthogonal polynomials: a functional approach, Acta Applicandae Mathematicae 34 (1994), no. 3, 283-303.

[5] B. G. Mertzios, Leverrier's algorithm for singular systems, IEEE Transactions on Automatic Control 29 (1984), no. 7, 652-653.

[6] G. Wang and L. Qiu, Leverrier-Chebyshev algorithm for the singular pencils, Linear Algebra and Its Applications 345 (2002), no. 1-3, 1-8.

Javier Hernández: Departamento de Matemáticas, Universidad Carlos III de Madrid, 28911 Leganés, Spain

E-mail address: jhbenite@math.uc3m.es

Francisco Marcellán: Departamento de Matemáticas, Universidad Carlos III de Madrid, 28911 Leganés, Spain

E-mail address: pacomarc@ing.uc3m.es 


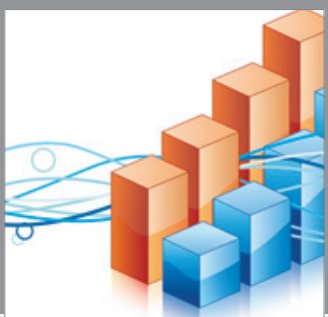

Advances in

Operations Research

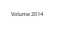

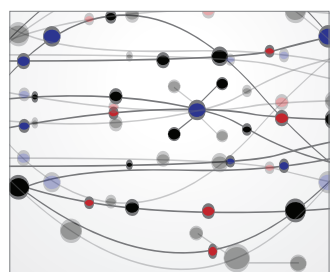

\section{The Scientific} World Journal
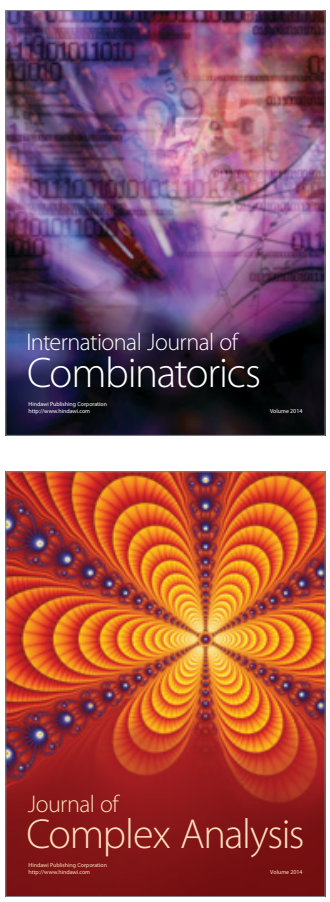

International Journal of

Mathematics and

Mathematical

Sciences
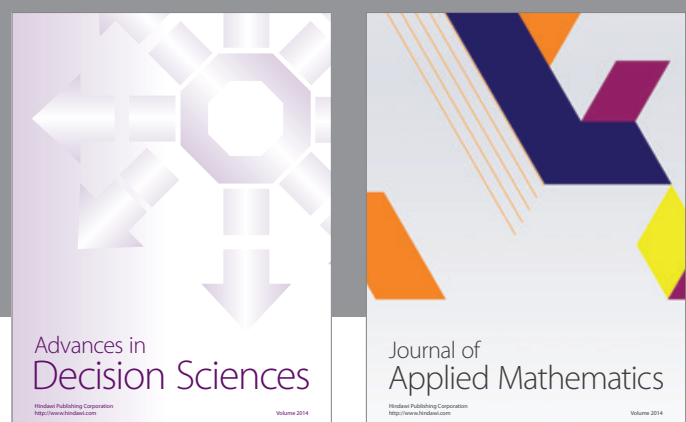

Journal of

Applied Mathematics
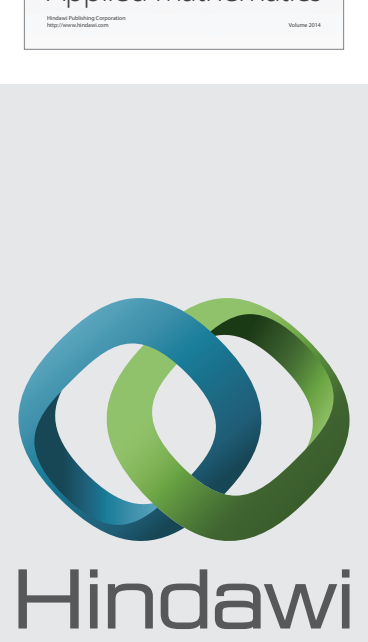

Submit your manuscripts at http://www.hindawi.com
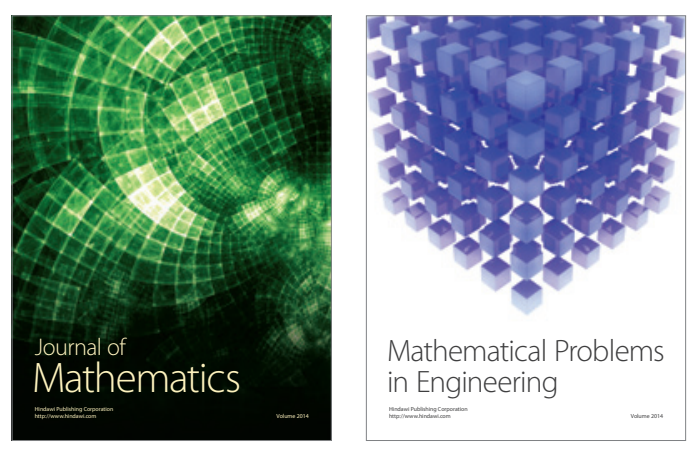

Mathematical Problems in Engineering
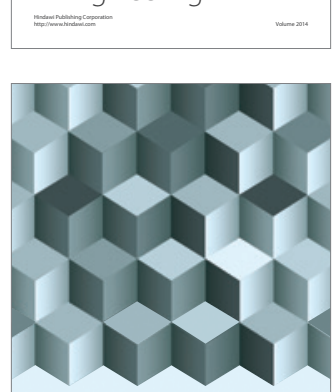

Journal of

Function Spaces
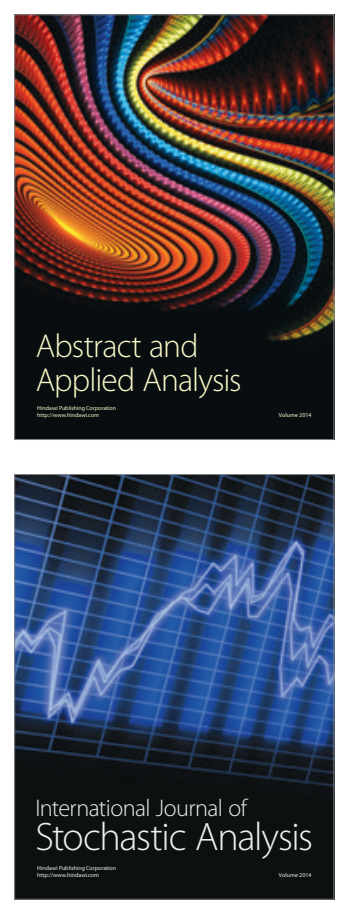

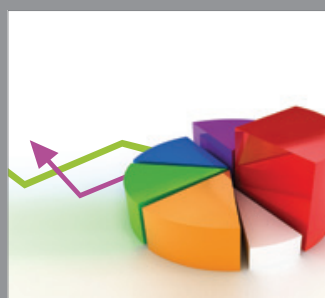

ournal of

Probability and Statistics

Promensencen
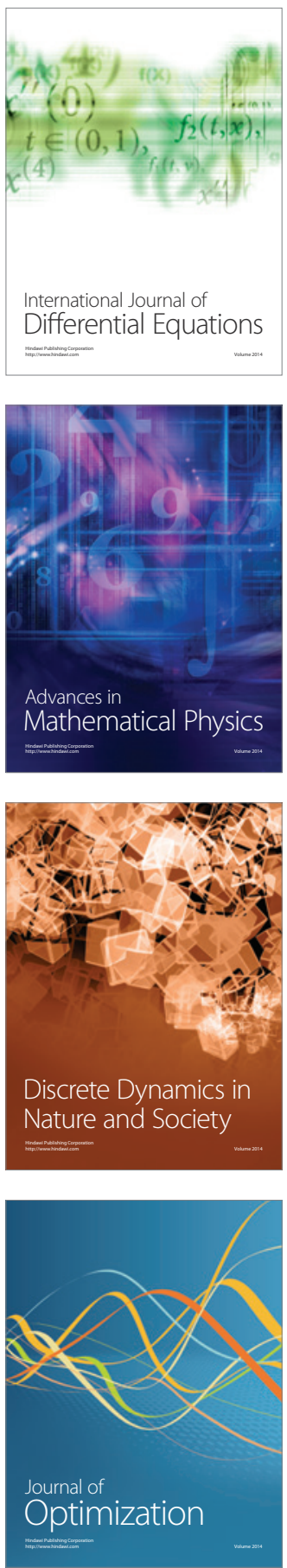